\title{
Progression-free survival as a potential surrogate for overall survival in metastatic breast cancer
}

This article was published in the following Dove Press journal:

OncoTargets and Therapy

18 June 2014

Number of times this article has been viewed

\section{Catherine Beauchemin' \\ Dan Cooper ${ }^{2}$ \\ Marie-Ėve Lapierre' \\ Louise Yelle ${ }^{3}$ \\ Jean Lachaine'}

'Université de Montréal, Faculté de pharmacie, Montreal, ${ }^{2}$ Institut national d'excellence en santé et en services sociaux (INESSS), ${ }^{3}$ Centre Hospitalier de l'Université de Montréal - Hôpital Notre-Dame, Département de médecine, Université de Montréal, Montreal, QC, Canada
Correspondence: Catherine Beauchemin Faculty of Pharmacy, University of Montreal, PO Box 6I28, Station Centreville, Montreal, QC, H3C 3J7, Canada

$\mathrm{Tel}+\mathrm{I} 514343611 \mathrm{I}$ ext 0717

Fax + I 5143432102

Email catherine.beauchemin@umontreal.ca
Background: Progression-free survival (PFS) and time to progression (TTP) are frequently used to establish the clinical efficacy of anti-cancer drugs. However, the surrogacy of PFS/TTP for overall survival (OS) remains a matter of uncertainty in metastatic breast cancer (mBC). This study assessed the relationship between PFS/TTP and OS in $\mathrm{mBC}$ using a trial-based approach.

Methods: We conducted a systematic literature review according to the PICO method: 'Population' consisted of women with mBC; 'Interventions' and 'Comparators' were standard treatments for $\mathrm{mBC}$ or best supportive care; 'Outcomes' of interest were median PFS/TTP and OS. We first performed a correlation analysis between median PFS/TTP and OS, and then conducted subgroup analyses to explore possible reasons for heterogeneity. Then, we assessed the relationship between the treatment effect on PFS/TTP and OS. The treatment effect on PFS/ TTP and OS was quantified by the absolute difference of median values. We also conducted linear regression analysis to predict the effects of a new anti-cancer drug on OS on the basis of its effects on PFS/TTP.

Results: A total of 5,041 studies were identified, and 144 fulfilled the eligibility criteria. There was a statistically significant relationship between median PFS/TTP and OS across included trials $(\mathrm{r}=0.428 ; P<0.01)$. Correlation coefficient for the treatment effect on PFS/TTP and OS was estimated at $0.427(P<0.01)$. The obtained linear regression equation was $\Delta \mathrm{OS}=-0.088(95 \%$ confidence interval $[\mathrm{CI}]-1.347-1.172)+1.753\left(95 \%\right.$ CI 1.307-2.198) $\times \Delta \mathrm{PFS}\left(\mathrm{R}^{2}=0.86\right)$.

Conclusion: Results of this study indicate a significant association between PFS/TTP and OS in $\mathrm{mBC}$, which may justify the use of PFS/TTP in the approval for commercialization and reimbursement of new anti-cancer drugs in this cancer setting.

Keywords: progression-free survival, time to progression, surrogate endpoint, metastatic breast cancer

\section{Introduction}

Improvement in overall survival (OS) has historically been considered the gold standard for assessing clinical benefits of a new anti-cancer drug in the context of advanced cancers. OS, defined as the time from randomization to time of death from any cause, represents a universally accepted endpoint given its objectivity, its clinical relevance, and its ease of interpretation. ${ }^{1,2}$ However, OS may be affected by the use of subsequent-line therapies after disease progression, which makes it difficult to assess the impact of only one treatment on survival. OS may also be influenced by the confounding effect of crossover therapy, as many clinical trials allow patients in the control arm to receive the experimental treatment after disease progression for ethical reasons. Moreover, OS requires trials with an extended follow-up period, which, as a 
result, leads to a substantial increase in costs and time before approval of a new anti-cancer drug. ${ }^{1,3,4}$

In an effort to overcome limitations associated with OS, surrogate endpoints based on tumor assessment have been accepted as prognostic indicators of clinical benefits for drug approval. ${ }^{5}$ During the past decades, several anti-cancer drugs have been approved for commercialization based on surrogate endpoints other than OS. ${ }^{6,7}$ Progression-free survival (PFS), defined as the time from randomization to objective tumor progression or death, and time to progression (TTP), defined as the time from randomization to objective tumor progression only, are commonly used alternate endpoints for OS in clinical trials of advanced cancers. According to the US Food and Drug Administration (FDA), PFS represents a preferred regulatory endpoint because it includes deaths due to tumor progression or adverse drug events. ${ }^{8}$ PFS and TTP are attractive alternative endpoints for clinical trials because they are not influenced by subsequent or crossover therapies received after disease progression, they are generally based on objective and quantitative assessment, and they require smaller sample size and shorter follow-up periods to detect significant differences between treatments compared with OS trials. ${ }^{1,8}$ However, these endpoints are subject to assessment bias, especially in openlabel trials, and, more importantly, they are not validated as adequate surrogates for OS in all cancer settings. ${ }^{8}$

According to Hughes, ${ }^{9}$ there is a hierarchy of information to consider when validating a potential surrogate endpoint:

1) Establish a biological rationale for potential surrogacy, 2) show the prognostic value of the surrogate endpoint in the absence and 3) the presence of treatments for the disease of interest and 4) show the association between the differences in the effect of randomized treatments on the potential surrogate endpoint and the differences in the effect on the clinical endpoint. ${ }^{9}$

During the past years, many efforts have been made to validate PFS as a surrogate endpoint for OS in different cancer settings, including advanced colorectal cancer, advanced breast cancer, advanced non-small-cell lung cancer, advanced ovarian cancer, non-Hodgkin's lymphoma, advanced gastric cancer, glioblastoma multiforme, and metastatic prostate cancer. ${ }^{10,11}$ Although the surrogacy of PFS for OS has been established in advanced colorectal cancer, the validation of this surrogate endpoint remains a matter of controversy in other cancer settings, such as in metastatic breast cancer $(\mathrm{mBC}) .{ }^{12}$ Therefore, the aim of the present study is to assess the relationship between PFS/TTP and OS in mBC using a trial-based approach.

\section{Materials and methods Systematic literature review}

A systematic review of the literature was conducted according to the PICO method: 'Population' consisted of women with $\mathrm{mBC}$; 'Interventions' and 'Comparators' were standard treatments for $\mathrm{mBC}$ or best supportive care; 'Outcomes' of interest were median PFS and median OS. A structured literature search was performed using electronic databases such as MEDLINE (1950-2010), EMBASE (1980-2010), all evidence-based medicine reviews (including Cochrane Database of Systematic Reviews, American College of Physician Journal Club, Database of Abstract of Reviews of Effects, Cochrane Controlled Trials Register, Cochrane Methodology Register, Health Technology Assessment Database, and National Health Service [NHS] Economic Evaluation Database), and Current Content (1993-2010). The following keywords were used for the search: breast cancer, breast carcinoma, breast neoplasm, metastasis, advanced cancer, advanced breast neoplasm, advanced tumor, survival, disease progression, cancer survival, survival time, survival rate, progression, progression-free survival, time to progression, time to disease progression, recurrence-free survival, event-free survival, cause specific survival, and survival analysis. Grey literature was searched in order to limit publication bias. More specifically, we reviewed clinical trials registries, abstracts from key annual meetings, and reports from regulatory agencies for relevance. Furthermore, reference lists of included articles and relevant systematic reviews were screened to identify additional publications.

The literature search was performed to identify randomized clinical trials of $\mathrm{mBC}$ therapy reporting both median PFS or TTP and median OS. The search was limited to studies with a sample size of at least 50 patients per treatment group that were published in English or French between January 1990 and April 2010. Studies were excluded if less than 80\% of the patient sample had a metastatic disease, if the treatment under investigation included surgery or radiotherapy, and if the full-text article was not available. All eligibility criteria were defined a priori.

Titles were initially screened for relevance. Abstracts of studies potentially meeting the eligibility criteria were then reviewed. Finally, full-text articles were obtained for studies deemed eligible for inclusion according to the abstract and were assessed using a pre-defined eligibility form. When more than one publication was retrieved for the same trial, we selected the most recent article. For each study selected for inclusion, the following information was extracted: first author, year of publication, sample size, median PFS or 
TTP, median OS, definition of surrogate endpoint, mean age, median follow-up, type of treatment under investigation, prior treatments for $\mathrm{mBC}$, estrogen receptor (ER), progesterone receptor (PR), and human epidermal growth factor receptor (HER)-2 status, menopausal status, performance score, metastatic sites, and number of metastatic sites. The allowance for patients in the control arm to crossover to the experimental treatment after progression was also extracted. Data extraction was performed according to the intention-to-treat population. Study selection and data extraction were performed by two independent reviewers in order to ensure appropriate validation. Disagreement between reviewers was discussed and resolved by consensus. Study quality was assessed using the Jadad scale, a widely used tool for evaluating the methodological quality of clinical trials. ${ }^{13}$

\section{Statistical analysis}

The validation of PFS/TTP as a surrogate endpoint for OS in $\mathrm{mBC}$ relies on two main conditions. First, both PFS/TTP and OS endpoints must be correlated, and second, the treatment effect on PFS/TTP and OS must also be correlated. Once these conditions are fulfilled, a prediction model for the effects of a new anti-cancer drug on OS based on its effects on PFS/TTP can be built.

In the present study, a correlation analysis between PFS/TTP and OS was first performed to determine if these measures could be considered as valid surrogate endpoints for OS. For this analysis, each treatment arm provided one observation. All observations were tested for normality using the Kolmogorov-Smirnov test. The relationship between PFS/TTP and OS was evaluated using Pearson's productmoment correlation or Spearman's rank correlation, depending on whether observations followed a normal distribution. Pearson's or Spearman's correlation coefficients were calculated to examine the degree of association between both measures of survival. Degrees of association were defined a priori; correlation coefficients ranging from 0.00 to $0.19,0.20$ to $0.39,0.40$ to $0.59,0.60$ to 0.79 , and superior to 0.8 were considered to represent a very weak, weak, moderate, strong, and very strong association, respectively. ${ }^{14}$ Subgroup analyses were also conducted to explore possible reasons for heterogeneity. The relationship between the treatment effect on PFS/TTP and the treatment effect on OS was then assessed. For this analysis, each study was analyzed as a unit. The treatment effect on PFS/TTP and OS was measured in terms of the absolute difference of medians. For studies that included more than two treatment arms, the experimental arm was compared with a randomly chosen control arm within the same study in order to avoid the analysis of correlated data. Pearson's or Spearman's correlation coefficients were calculated to evaluate the degree of association between the treatment effect on PFS/TTP ( $\Delta \mathrm{PFS} / \Delta \mathrm{TTP})$ and OS ( $\Delta \mathrm{OS})$, according to the normality of the data.

Linear regression analysis was conducted in an attempt to predict the effects of a new anti-cancer drug on OS on the basis of its effects on PFS/TTP. For this analysis, only studies that presented a statistically significant difference in both PFS/TTP and OS between treatment arms were used in order to build a prediction model, minimizing the potential bias due to the influence of subsequent or crossover therapy after progression. The coefficient of determination $\left(\mathrm{R}^{2}\right)$ was calculated to measure the proportion of variance in $\Delta$ OS explained by the variance in $\Delta \mathrm{PFS} / \Delta \mathrm{TTP}$.

For all statistical analyses, OS was defined as the time from randomization to time of death from any cause, whereas PFS and TTP were defined as the time from randomization to objective tumor progression or death and the time from randomization to objective tumor progression, respectively. Parametric statistical analyses were weighted by trial sample size. All statistical analyses were performed using IBM SPSS Statistics (version 19) (IBM, Armonk, NY, USA). Statistic tests were two-sided, and levels of significance were 0.05 .

\section{Results}

\section{Trials included in the analysis}

A total of 5,041 potentially relevant studies were identified through the literature search, of which 1,356 full-text articles were assessed using a pre-defined eligibility form. Of these studies, 142 fulfilled the eligibility criteria and two additional relevant studies were retrieved through the grey literature search and cross-referencing, leading to an inclusion of 144 studies (Figure 1, Supplementary materials). Selected studies included 315 treatment arms, which represents 43,459 patients with $\mathrm{mBC}$.

A detailed description of study characteristics is presented in Table 1. In brief, mean patient age ranged from 41 to 69 years, with a majority of patients aged from 50 to 59 years. The type of therapy under investigation was mostly chemotherapy, used as monotherapy or as a combination therapy. Biological therapy was assessed in approximately $10 \%$ of included studies. More specifically, anti-HER2 therapy was given in all studies that included patients with a confirmed positive HER2 expression. In most selected studies, included patients were postmenopausal and had a favorable performance status. Overall, the methodological 


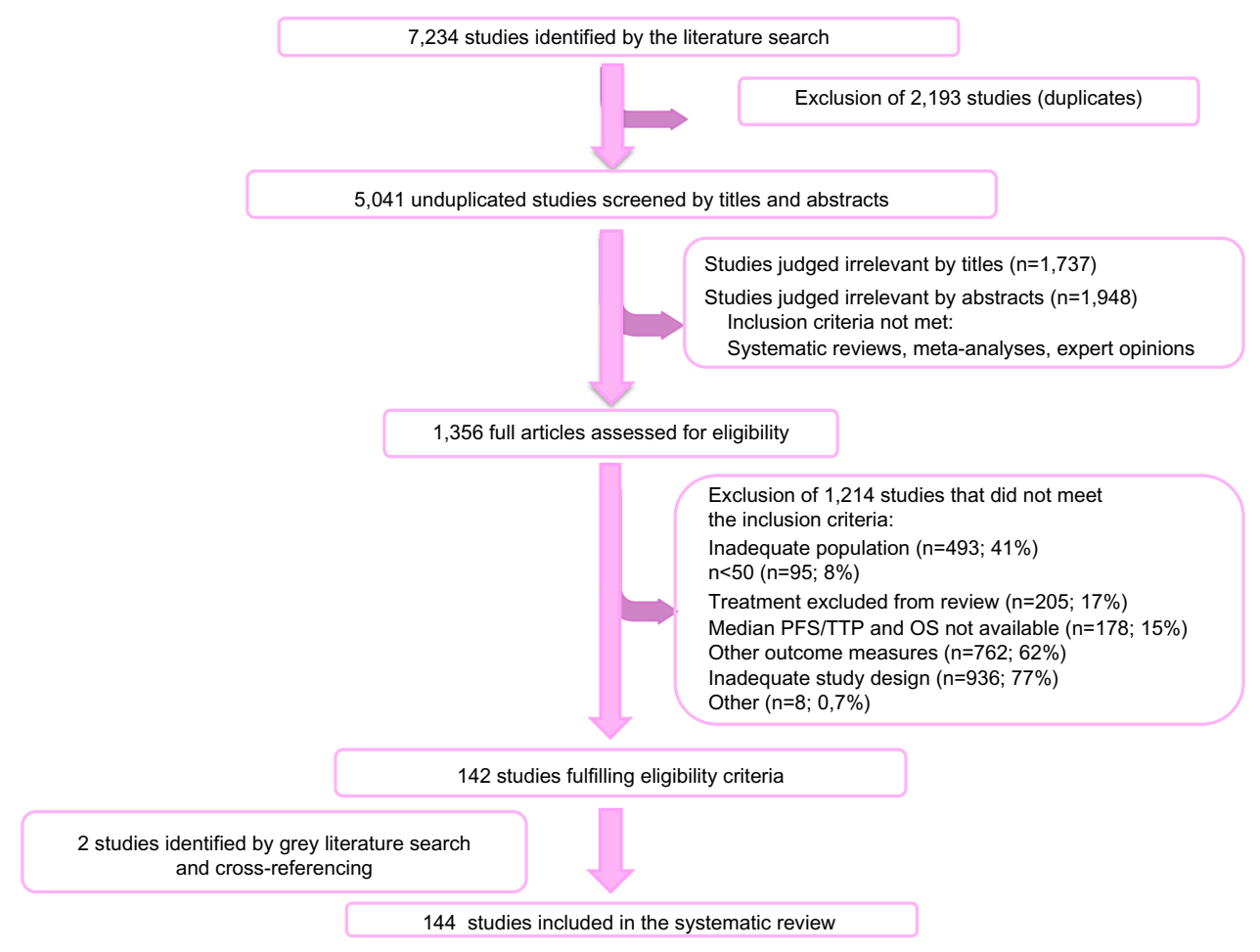

Figure I Flow chart of studies included in the systematic review of the literature.

Abbreviations: OS, overall survival; PFS, progression-free survival; TTP, time to progression.

quality of included studies was considered good, as more than $70 \%$ of studies had a Jadad score $\geq 3$.

\section{Correlation between median PFS/TTP and median OS}

There was a significant relationship between PFS/TTP and OS across trials (Figure 2). The unweighted Spearman correlation coefficient for the PFS/TTP and the OS was 0.428 ( $P \leq 0.01)$, which corresponds to a moderate association according to pre-defined criteria. Results of subgroup analyses are presented in Table 2 . The correlation between PFS/TTP and OS was higher for studies published after year 2000 ( $\mathrm{r}=0.537 ; P \leq 0.01$ ). Moreover, analyses according to the definition of surrogate endpoint showed that the correlation was higher for studies using PFS as an endpoint $(\mathrm{r}=0.523$; $P \leq 0.01)$ than for those using TTP $(\mathrm{r}=0.307 ; P \leq 0.01)$, which may be explained by the inclusion of deaths in the PFS definition. Analysis of the impact of type of therapy under investigation showed a greater association between PFS/TTP and OS in studies evaluating chemotherapy single agent $(\mathrm{r}=0.575 ; P \leq 0.01)$ or in combination $(\mathrm{r}=0.632$; $P \leq 0.01$ ) than in those evaluating hormone therapy (nonsignificant r). A statistically significant correlation was found in studies evaluating combination with a biological therapy ( $\mathrm{r}=0.662 ; P \leq 0.01)$, even if analysis was performed on a small number of studies. Moreover, correlations tended to be higher in studies assessing subsequent-line therapy, as higher correlation coefficients were obtained especially when prior chemotherapy for $\mathrm{mBC}$ was allowed ( $\mathrm{r}=0.596 ; P \leq 0.01)$. Lastly, correlations were greater for studies with a predominance of patients with visceral metastases $(\mathrm{r}=0.547 ; P \leq 0.01)$ or three or more metastatic sites $(\mathrm{r}=0.729 ; P \leq 0.01)$.

\section{Correlation between treatment effect on PFS/TTP and OS}

For the correlation analysis between treatment effect on PFS/ TTP and OS, pair-wise treatment comparisons from each study were analyzed as a unit. A total of 149 pair-wise treatment comparisons were included for analysis when multiplearm trials were considered. A significant relationship between differences in median PFS/TTP and differences in median OS was seen within trials. The unweighted Spearman correlation coefficient was estimated at $0.427(P \leq 0.01)$, corresponding to a moderate association according to pre-defined criteria.

\section{Regression analysis between treatment effect on PFS/TTP and OS}

In total, 14 studies presented a statistically significant difference in both PFS/TTP and OS between treatment arms. Results of the linear regression analysis indicated 
Table I Characteristics of studies included in the analysis

\begin{tabular}{|c|c|}
\hline Characteristics of selected studies & $\begin{array}{l}\% \text { of } \\
\text { selected } \\
\text { studies }\end{array}$ \\
\hline \multicolumn{2}{|l|}{ Year of publication } \\
\hline $1990-1994$ & 16.0 \\
\hline $1995-1999$ & 25.0 \\
\hline $2000-2004$ & 28.5 \\
\hline $2005-2010$ & 30.6 \\
\hline \multicolumn{2}{|l|}{ Definition of surrogate endpoint } \\
\hline PFS & 49.3 \\
\hline TTP & 49.3 \\
\hline Inconsistent definition & 1.4 \\
\hline \multicolumn{2}{|l|}{ Possibility for crossover after progression } \\
\hline Yes & 37.5 \\
\hline No & 19.4 \\
\hline $\begin{array}{l}\text { Subsequent therapy after progression given } \\
\text { at physician's discretion }\end{array}$ & 6.9 \\
\hline Not reported & 36.1 \\
\hline \multicolumn{2}{|l|}{ Type of therapy under investigation } \\
\hline Chemotherapy (monotherapy) & 20.1 \\
\hline Hormone therapy (monotherapy) & 15.3 \\
\hline Biological therapy (monotherapy)* & 2.1 \\
\hline Anti-HER2 therapy & 2.1 \\
\hline Anti-VEGF therapy & 0 \\
\hline Combination with chemotherapy & 43.1 \\
\hline Combination including hormone therapy & II.I \\
\hline Combination including biological therapy* & 8.3 \\
\hline Anti-HER2 combination & 0.7 \\
\hline Anti-HER2 plus chemotherapy & 4.9 \\
\hline Anti-HER2 plus hormone therapy & 1.4 \\
\hline Anti-VEGF plus chemotherapy & 1.4 \\
\hline \multicolumn{2}{|l|}{ Prior chemotherapy for metastatic disease allowed } \\
\hline Yes & 29.9 \\
\hline No & 61.8 \\
\hline Not reported & 8.3 \\
\hline \multicolumn{2}{|l|}{ Prior hormone therapy for metastatic disease allowed } \\
\hline Yes & 52.8 \\
\hline No & II.I \\
\hline Not reported & 36.1 \\
\hline \multicolumn{2}{|l|}{ Mean patient age (years) } \\
\hline $40-49$ & 5.6 \\
\hline $50-59$ & 64.6 \\
\hline $60-69$ & 23.6 \\
\hline Mean age not reported & 6.3 \\
\hline \multicolumn{2}{|l|}{$\mathrm{mBC}$ phenotypes based on ER/PR receptor status } \\
\hline Studies with a predominance of patients with ER/PR+** & 38.9 \\
\hline Studies with a predominance of patients with ER-/PR- & 1.4 \\
\hline $\begin{array}{l}\text { Studies with a predominance of patients with ER/PR } \\
\text { unknown }\end{array}$ & 9.7 \\
\hline ER/PR status not reported & 50.0 \\
\hline \multicolumn{2}{|l|}{$\begin{array}{l}\mathrm{mBC} \text { phenotypes based on HER2, ER and PR } \\
\text { receptor status }\end{array}$} \\
\hline Studies with HER2+, ER/PR any*** as a selection criteria & 6.9 \\
\hline $\begin{array}{l}\text { Studies with a predominance of patients with } \\
\text { HER2+, ER/PR+** }\end{array}$ & 3.5 \\
\hline $\begin{array}{l}\text { Studies with a predominance of patients with } \\
\text { HER2+, ER-/PR- }\end{array}$ & 1.4 \\
\hline $\begin{array}{l}\text { Studies with a predominance of patients with } \\
\text { HER2-, ER/PR any*** }\end{array}$ & 5.6 \\
\hline
\end{tabular}

(Continued)
Table I (Continued)

\begin{tabular}{|c|c|}
\hline Characteristics of selected studies & $\begin{array}{l}\% \text { of } \\
\text { selected } \\
\text { studies }\end{array}$ \\
\hline $\begin{array}{l}\text { Studies with a predominance of patients with } \\
\text { HER2-, ER/PR+** }\end{array}$ & 4.9 \\
\hline $\begin{array}{l}\text { Studies with a predominance of patients with } \\
\text { HER2-, ER-/PR- }\end{array}$ & 0.0 \\
\hline HER2, ER/PR status not reported & 86.1 \\
\hline \multicolumn{2}{|l|}{ Menopausal status } \\
\hline $\begin{array}{l}\text { Studies with a predominance of patients with } \\
\text { premenopausal status }\end{array}$ & 2.8 \\
\hline $\begin{array}{l}\text { Studies with a predominance of patients with } \\
\text { peri or postmenopausal status }\end{array}$ & 57.6 \\
\hline Menopausal status not reported & 39.6 \\
\hline \multicolumn{2}{|l|}{ ECOG performance score } \\
\hline $\begin{array}{l}\text { Studies with a predominance of patients with } \\
\text { ECOG score } 0\end{array}$ & 37.5 \\
\hline $\begin{array}{l}\text { Studies with a predominance of patients with } \\
\text { ECOG score I }\end{array}$ & 27.1 \\
\hline $\begin{array}{l}\text { Studies with a predominance of patients with } \\
\text { ECOG score 0-I; as reported }\end{array}$ & 6.9 \\
\hline ECOG performance score not reported & 28.5 \\
\hline \multicolumn{2}{|l|}{ Dominant metastatic site } \\
\hline $\begin{array}{l}\text { Studies with a predominance of patients with bone } \\
\text { metastases as a dominant site }\end{array}$ & 13.2 \\
\hline $\begin{array}{l}\text { Studies with a predominance of patients with soft } \\
\text { tissue metastases as a dominant site }\end{array}$ & 9.0 \\
\hline $\begin{array}{l}\text { Studies with a predominance of patients with visceral } \\
\text { metastases as a dominant site }\end{array}$ & 69.4 \\
\hline $\begin{array}{l}\text { Studies with a predominance of patients with another } \\
\text { dominant site }\end{array}$ & 0.7 \\
\hline Dominant metastatic site not reported & 7.6 \\
\hline \multicolumn{2}{|l|}{ Number of metastatic sites } \\
\hline $\begin{array}{l}\text { Studies with a predominance of patients with one } \\
\text { metastatic site }\end{array}$ & 12.5 \\
\hline $\begin{array}{l}\text { Studies with a predominance of patients with two } \\
\text { metastatic sites }\end{array}$ & 22.9 \\
\hline $\begin{array}{l}\text { Studies with a predominance of patients with three } \\
\text { or more metastatic sites }\end{array}$ & 16.0 \\
\hline Number of metastatic sites not reported & 48.6 \\
\hline \multicolumn{2}{|l|}{ Jadad score } \\
\hline Jadad score I & 0.7 \\
\hline Jadad score 2 & 28.5 \\
\hline Jadad score $\geq 3$ & 70.8 \\
\hline
\end{tabular}

Notes: *Biological therapy includes anti-HER2 therapy (trastuzumab, lapatinib or canertinib) and anti-VEGF therapy (bevacizumab); **ER/PR+ is defined as ER+/PR+, $E R+/ P R-$, and $E R-/ P R+; * * * E R / P R$ any includes $E R+/ P R+, E R+/ P R-$ and $E R-/ P R+$, $E R-/ P R$ - and ER/PR unknown.

Abbreviations: ECOG, Eastern Cooperative Oncology Group; ER, estrogen receptor; HER2, human epidermal growth factor receptor 2; $\mathrm{mBC}$, metastatic breast cancer; PFS, progression-free survival; PR, progesterone receptor; TTP, time to progression; VEGF, vascular endothelial growth factor.

a strong association between differences in median PFS/ TTP and differences in median OS (Figure 3). The regression equation was $\Delta \mathrm{OS}=-0.088(95 \%$ confidence interval $[\mathrm{CI}]-1.347-1.172)+1.753(95 \% \mathrm{CI} 1.307-2.198) \times \Delta \mathrm{PFS} /$ TTP, with a proportion of variation explained $\left(\mathrm{R}^{2}\right)$ of 0.86 . 


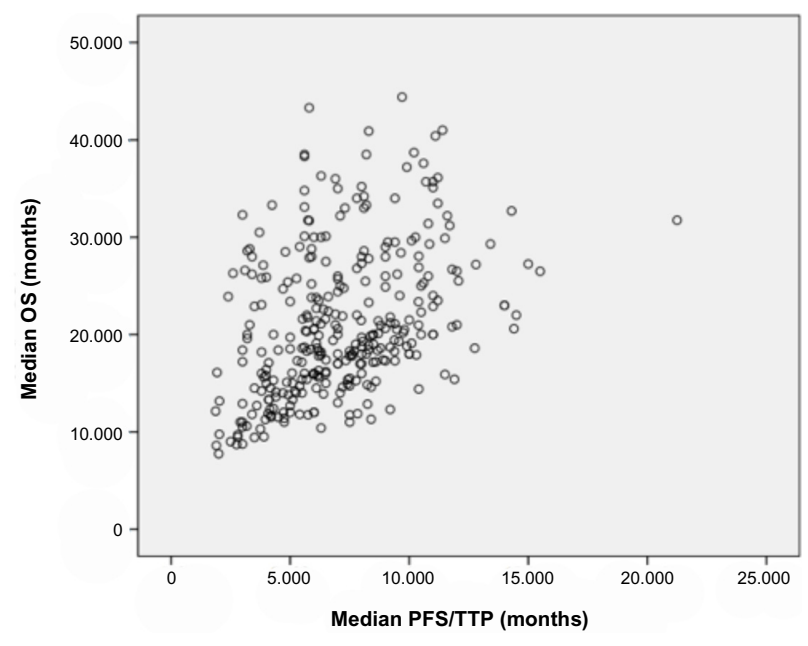

Figure 2 Correlation between median progression-free survival (months) and median overall survival (months) in metastatic breast cancer.

Note: Each circle corresponds to a treatment arm.

Abbreviations: OS, overall survival; PFS, progression-free survival; TTP, time to progression.

For example, results of the regression analysis predict that a difference in median PFS/TTP of 5, 10, 15, and 20 months would translate into a difference in median OS of 8.7, 17.4, 26.2, and 35.0 months, respectively.

\section{Discussion}

Over past decades, several new anti-cancer drugs have shown promising results in terms of efficacy by extending PFS or TTP in patients with $\mathrm{mBC}$. However, it becomes increasingly difficult to demonstrate the clinical benefits of a new anti-cancer drug in terms of OS, possibly due to the confounding effect of the subsequent or crossover therapy after progression and the substantial loss of follow-up, leading to low statistical power to detect clinically relevant differences in OS. Moreover, use of PFS or TTP remains an important challenge in the economic evaluation of anti-cancer drugs. From an economic standpoint, OS is considered as a preferred efficacy measure because it enables the conduct of costeffectiveness analyses and cost-utility analyses expressed in terms of cost per life-year gained (LYG) and cost per quality-adjusted life-year (QALY), respectively. Therefore, studies assessing the relationship between PFS/TTP and OS were needed to confirm that this endpoint is a valid surrogate for OS, thus justifying its use in the reimbursement of new oncology drugs and allowing better access to effective anticancer therapies.

Validating PFS as a surrogate endpoint for OS in advanced cancer remains a substantial challenge. In fact, several factors may significantly affect the correlation between PFS and OS, especially the number of effective subsequent regimens and the possibility of crossover to the investigational therapy after disease progression. According to Broglio and Berry, ${ }^{15}$ who evaluated the impact of postprogression survival (PPS) on the surrogacy of PFS for OS, the correlation between PFS and OS becomes hardly reliable as PPS lengthens. Therefore, the availability of effective subsequent treatments after disease progression plays an important role in the association between both endpoints, because a long post-progression period adds randomness that attenuates the ability to detect OS benefits. This was observed in the present analysis, as the correlation between PFS/TTP and OS was stronger in studies assessing secondline and later chemotherapy. Moreover, because crossover from control arm to the investigational arm might reduce the difference in OS between study arms, the predefined sample size of studies allowing crossover at disease progression might become insufficient to statistically detect the reduced difference in OS. As opposed to the extraction of other parameters that left no room for interpretation, the extraction of data on crossover from control arm to the investigational arm was based on subjective assessment. Indeed, few trials clearly reported the possibility for crossover therapy in the description of the study design. Therefore, to avoid a misleading analysis, correlation between PFS and OS according to crossover status was not assessed.

The assessment of the relationship between PFS/TTP and $\mathrm{OS}$ in $\mathrm{mBC}$ is highly relevant considering the substantial incidence of the disease and the large number of anti-cancer drugs approved for this indication over recent years. Moreover, $\mathrm{mBC}$ is associated with a limited life expectancy, with only one-fifth of patients surviving up to 5 years. ${ }^{16}$ Because the duration of PPS has been proven to greatly impact the surrogacy of PFS/TTP for OS, it was even more relevant to assess the relationship between endpoints in the metastatic setting, as opposed to an earlier stage of the disease. According to a literature review by Saad et al, ${ }^{4}$ the average median PPS observed in advanced breast cancer trials was estimated at 13.6 months, with significantly longer PPS in hormone therapy trials than in chemotherapy trials (20.5 vs 11.4 months, respectively; $P<0.001$ ). These relatively short durations of PPS therefore reinforce the significance of assessing the relationship between PFS/TTP and $\mathrm{OS}$ in the specific context of $\mathrm{mBC}$.

Based on previous studies, the surrogacy of PFS/TTP for OS in the context of $\mathrm{mBC}$ has not been clearly established. A meta-regression by Hackshaw et al, ${ }^{17}$ which included trials 
Table 2 Correlation analyses between median progression-free survival/time to progression and median overall survival according to characteristics of selected trials

\begin{tabular}{|c|c|c|c|}
\hline Characteristics of selected studies & $\begin{array}{l}\text { Number of } \\
\text { observations }\end{array}$ & $\begin{array}{l}\text { Spearman } \\
\text { correlation } \\
\text { coefficient }\end{array}$ & $P$-value \\
\hline \multicolumn{4}{|l|}{ Year of publication } \\
\hline $1990-1999$ & 130 & 0.248 & $\leq 0.01$ \\
\hline $2000-2010$ & 185 & 0.537 & $\leq 0.01$ \\
\hline \multicolumn{4}{|l|}{ Definition of surrogate endpoint } \\
\hline PFS & 154 & 0.523 & $\leq 0.01$ \\
\hline TTP & 157 & 0.307 & $\leq 0.01$ \\
\hline \multicolumn{4}{|l|}{ Type of therapy under investigation } \\
\hline Chemotherapy (monotherapy) & 80 & 0.575 & $\leq 0.01$ \\
\hline Hormone therapy (monotherapy) & 63 & 0.201 & NS \\
\hline Biological therapy (monotherapy) & 6 & 0.714 & NS \\
\hline Combination with chemotherapy & $|3|$ & 0.632 & $\leq 0.01$ \\
\hline Combination including hormone therapy & 21 & 0.391 & NS \\
\hline Combination including biological therapy & 14 & 0.662 & $\leq 0.01$ \\
\hline \multicolumn{4}{|c|}{ Prior chemotherapy for metastatic disease allowed } \\
\hline Yes & 92 & 0.596 & $\leq 0.01$ \\
\hline No & 198 & 0.310 & $\leq 0.01$ \\
\hline \multicolumn{4}{|c|}{ Prior hormone therapy for metastatic disease allowed } \\
\hline Yes & 169 & 0.346 & $\leq 0.01$ \\
\hline No & 35 & -0.015 & NS \\
\hline \multicolumn{4}{|l|}{ Mean patient age (years) } \\
\hline $40-59.9$ & 225 & 0.560 & $\leq 0.01$ \\
\hline $60-69.9$ & 71 & 0.377 & $\leq 0.01$ \\
\hline \multicolumn{4}{|c|}{$\mathrm{mBC}$ phenotypes based on ER/PR receptor status ${ }^{\dagger}$} \\
\hline $\mathrm{ER} / \mathrm{PR}+*$ & 125 & 0.346 & $\leq 0.01$ \\
\hline ER-/PR- & 3 & 1.000 & NS \\
\hline ER/PR unknown & 32 & 0.629 & $\leq 0.01$ \\
\hline \multicolumn{4}{|c|}{$\mathrm{mBC}$ phenotypes based on HER2, ER and PR receptor status ${ }^{\dagger}$} \\
\hline HER2+, ER/PR any** & 23 & 0.665 & $\leq 0.01$ \\
\hline HER2+, ER/PR+* & 10 & 0.418 & NS \\
\hline HER2+, ER-/PR- & 3 & 1.000 & NS \\
\hline HER2-, ER/PR any** & 16 & 0.726 & $\leq 0.01$ \\
\hline HER2-, ER/PR+* & 14 & 0.771 & $\leq 0.01$ \\
\hline HER2-, ER-/PR- & 0 & N/A & $\mathrm{N} / \mathrm{A}$ \\
\hline \multicolumn{4}{|l|}{ Menopausal status ${ }^{\dagger}$} \\
\hline Premenopausal status & 8 & 0.167 & NS \\
\hline Peri or postmenopausal status & 169 & 0.347 & $\leq 0.01$ \\
\hline \multicolumn{4}{|l|}{ ECOG performance score ${ }^{\dagger}$} \\
\hline ECOG score 0 & 114 & 0.334 & $\leq 0.01$ \\
\hline ECOG score I & 90 & 0.385 & $\leq 0.01$ \\
\hline \multicolumn{4}{|l|}{ Dominant metastatic site ${ }^{\dagger}$} \\
\hline Bone metastases as a dominant site & 44 & -0.060 & NS \\
\hline Soft tissue metastases as a dominant site & 21 & 0.114 & NS \\
\hline Visceral metastases as a dominant site & 217 & 0.547 & $\leq 0.01$ \\
\hline \multicolumn{4}{|l|}{ Number of metastatic sites ${ }^{\dagger}$} \\
\hline One metastatic site & 42 & 0.259 & NS \\
\hline Two metastatic sites & 68 & 0.183 & NS \\
\hline Three or more metastatic sites & 59 & 0.729 & $\leq 0.01$ \\
\hline
\end{tabular}

Notes: ${ }^{\dagger}$ Analyses were performed according to predominance in included studies, as described in Table I; *ER/PR+ is defined as ER+/PR+, ER+/PR- or ER-/PR+; **ER/PR any includes ER+/PR+, ER+/PR- and ER-/PR+, ER-/PR- and ER/PR unknown.

Abbreviations: ECOG, Eastern Cooperative Oncology Group; ER, estrogen receptor; HER2, human epidermal growth factor receptor 2; mBC, metastatic breast cancer; N/A, not available; NS, not significant; PFS, progression-free survival; PR, progesterone receptor; TTP, time to progression. 


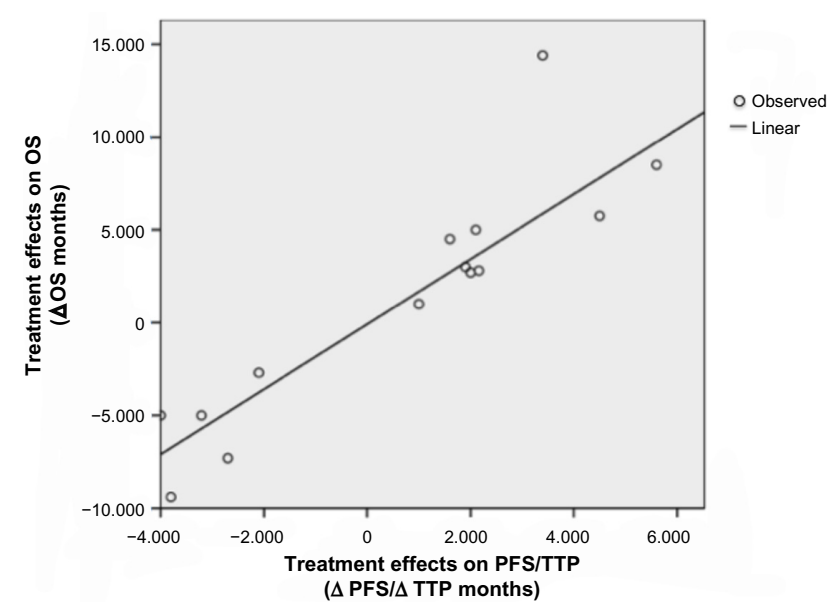

Figure 3 Linear relationship between the treatment effect on progression-free survival/time to progression ( $\Delta \mathrm{PFS} / \Delta \mathrm{TTP}$ ) and the treatment effect on overall survival ( $\triangle$ OS).

Note: Each study was analyzed as a unit.

Abbreviations: OS, overall survival; PFS, progression-free survival; TTP, time to progression; $\Delta$ OS, treatment effect on OS; $\Delta$ PFS, treatment effect on PFS; $\Delta$ TTP, treatment effect on TTP.

assessing first-line combination anthracycline chemotherapy for $\mathrm{mBC}$, reported a significant linear association between treatment effect on TTP and OS $\left(\mathrm{R}^{2}=0.56 ; P<0.001\right)$. Moreover, a positive relationship between progression and survival was reported in a trial-based analysis by Sherrill et al. ${ }^{18}$ Based on the results of 67 trials, these authors estimated an unweighted Pearson correlation coefficient between treatment effect on TTP (hazard ratio $[\mathrm{HR}]_{\mathrm{TTP}}$ ) and $\mathrm{OS}\left(\mathrm{HR}_{\mathrm{OS}}\right)$ at $0.46 .{ }^{18}$ A significant relationship between endpoints was also obtained in a meta-regression analysis including anthracycline or taxane-based chemotherapy trials. ${ }^{19}$ Despite the small number of included studies, $\mathrm{HR}_{\mathrm{PFS}}$ was shown to be a statistically significant predictor for $\mathrm{HR}_{\mathrm{OS}}$ for both anthracycline and taxanebased chemotherapy, with $\mathrm{R}^{2}$ of 0.49 and 0.35 , respectively. Burzykowski et $\mathrm{al}^{20}$ also found a moderate association between PFS/TTP and OS based on individual patient data from 11 randomized trials comparing an anthracycline with a taxane regimen as a first-line treatment for $\mathrm{mBC}$. However, the authors found a poor and imprecise relationship between treatment effects on PFS/TTP and OS and concluded that the relationship was insufficiently strong to claim surrogacy. ${ }^{20}$ Furthermore, Matsubara et $\mathrm{al}^{21}$ investigated the surrogacy of PFS for OS in a third-line setting for chemotherapy-resistant $\mathrm{mBC}$ and found no significant association between PFS and OS. However, the authors claim that this result may be due to the fact that, in the third-line treatment setting, OS almost overlaps PPS, which means that the treatment effects on PFS are too weak to affect OS. In brief, although previous studies have shown a tendency towards surrogacy of PFS for OS, published results remained controversial. The present analysis, which is distinguishable from previous work because of the exhaustiveness of study selection, confirms the previously reported tendency towards surrogacy.

An exhaustive and rigorous systematic literature review was conducted to retrieve clinical trials relevant for the assessment of the relationship between PFS/TTP and OS in $\mathrm{mBC}$. Indeed, several literature databases were consulted; an exhaustive search of the grey literature was performed to limit publication bias; study selection and data extraction were performed by two independent reviewers to ensure adequate validation; and study quality was assessed to illustrate variations among included studies. A 20 -year time period was covered in order to retrieve a large number of studies reporting both PFS/TTP and OS endpoints. Such a long timeframe provided a good overview of the relationship between endpoints. Moreover, a transparent methodology was used, which could be replicated to evaluate the surrogacy of PFS/TTP for OS in other cancer settings.

This study has several limitations. First, mostly due to the long timeframe of the present systematic review, differences in inclusion criteria and patient characteristics were observed across included trials, thus limiting the possibility to perform correlation analyses on a homogeneous group of patients. However, extensive subgroup correlation analyses were performed to identify potential sources of heterogeneity. Furthermore, definitions of PFS and TTP were not consistent across trials. All-cause mortality was often included in studies using TTP as a primary or secondary outcome, which by definition, should be done only for PFS outcome. Therefore, considering the heterogeneity of reported definitions, PFS and TTP outcomes were combined for the purpose of the analysis. However, this may have diluted the association between PFS/TTP and OS. Moreover, very few studies clearly described the use of subsequent treatments or crossover therapy after disease progression. Lack of detailed information on post-progression therapy may have had a substantial impact on the overall relationship between PFS/TTP and OS and on the regression model predicting the effects of a new anti-cancer therapy on OS based on its effects on PFS/TTP. In addition, parametric statistical analyses were weighted by trial sample size; however, non-parametric analyses such as Spearman's correlations were not weighted because of the impossibility to weight data converted to ranks. Nevertheless, an exploratory analysis indicated that very similar results were obtained when weighted and unweighted Pearson's correlation analyses were performed. Thus, the impact of not weighting Spearman's rank correlation analyses was 
considered minimal. Furthermore, in the present study, the relationship between PFS/TTP and OS was assessed using a trial-based approach. This approach has been proposed by several authors and has been largely used in the assessment of surrogate endpoints. ${ }^{22-24}$ Because this method takes into account the results of several clinical trials instead of a single trial, it provides high statistical power to detect a potential surrogate endpoint. However, when a trial-based approach is taken, the individual patient data are usually not available, thus limiting the possibility of assessing the surrogacy of an endpoint accounting for potential confounding variables such as family history, smoking status, and exercise level. Buyse et $\mathrm{al}^{25}$ proposed that the validation of a surrogate endpoint should be based, on the one hand, at the individual level, and on the other hand, at the trial level. In other words, they suggested the assessment of the correlation between endpoints at the individual level and the correlation between treatment effect on these endpoints at the trial level. Because individual patient data were not available for the completion of this study, the former analysis could not be performed. Therefore, an analysis taking into account information about patients that may act as potential confounders was not feasible. However, in the current analysis, only randomized controlled trials were included, which minimizes the impact of potential confounders on the results. Although the trial-based approach remains a method of choice to validate a surrogate endpoint, further assessment of the association between PFS/TTP and OS using individual patient data would be of great interest in order to ensure a thorough validation.

\section{Conclusion}

Findings of this study may have important clinical implications for the treatment of $\mathrm{mBC}$. The present work points toward a statistically significant relationship between PFS/ TTP and OS in the context of $\mathrm{mBC}$. This may justify the use of PFS/TTP in the approval for commercialization and reimbursement of new anti-cancer drugs in $\mathrm{mBC}$, thus allowing better access to effective anti-cancer drugs and ultimately improving patient survival.

\section{Acknowledgments}

The authors thank Marc Dorais for biostatistical support. The authors would also like to acknowledge the significant contribution of Nathalie Letarte.

\section{Disclosure}

The authors have no financial conflicts of interest. This work was supported by the Institut national d'excellence en santé et en services sociaux (INESSS), the Canadian Institutes for Health Research (CIHR), and the Fonds de recherche en santé du Québec (FRSQ). This study was conducted independently of the sponsor as part of a doctoral research project. The funding sources had no role in the study design, in the collection, analysis, and interpretation of the data, or in the writing or submission of the manuscript.

\section{References}

1. Zhuang SH, Xiu L, Elsayed YA. Overall survival: a gold standard in search of a surrogate: the value of progression-free survival and time to progression as end points of drug efficacy. Cancer J. 2009;15(5): 395-400.

2. Driscoll JJ, Rixe O. Overall survival: still the gold standard: why overall survival remains the definitive end point in cancer clinical trials. Cancer J. 2009;15(5):401-405.

3. Lebwohl D, Kay A, Berg W, Baladi JF, Zheng J. Progression-free survival: gaining on overall survival as a gold standard and accelerating drug development. Cancer J. 2009;15(5):386-394.

4. Saad ED, Katz A, Buyse M. Overall survival and post-progression survival in advanced breast cancer: a review of recent randomized clinical trials. J Clin Oncol. 2010;28(11):1958-1962.

5. Saad ED. Endpoints in advanced breast cancer: methodological aspects and clinical implications. Indian J Med Res. 2011;134:413-418.

6. Sridhara R, Johnson JR, Justice R, Keegan P, Chakravarty A, Pazdur R. Review of oncology and hematology drug product approvals at the US Food and Drug Administration between July 2005 and December 2007. J Natl Cancer Inst. 2010;102(4):230-243.

7. Johnson JR, Williams G, Pazdur R. End points and United States Food and Drug Administration approval of oncology drugs. J Clin Oncol. 2003;21(7):1404-1411.

8. US Department of Health and Human Services; Food and Drug Administration; Center for Drug Evaluation and Research (CDER); Center for Biologics Evalation and Research (CBER). Guidance for Industry. Clinical Trial Endpoints for the Approval of Cancer Drugs and Biologics. Rockville (MD): Food and Drug Administration(FDA); 2007. Available from: http://www.fda.gov/downloads/Drugs/.../Guidances/ ucm071590.pdf. Accessed March 30, 2012.

9. Hughes MD. Practical issues arising in an exploratory analysis evaluating progression-free survival as a surrogate endpoint for overall survival in advanced colorectal cancer. Stat Methods Med Res. 2008;17(5):487-495.

10. Sherrill B, Kaye JA, Sandin R, Cappelleri JC, Chen C. Review of metaanalyses evaluating surrogate endpoints for overall survival in oncology. Onco Targets Ther. 2012;5:287-296.

11. Davis S, Tappenden P, Cantrell A. A Review of Studies Examining the Relationship between Progression-Free Survival and Overall Survival in Advanced or Metastatic Cancer. Sheffield: Decision Support Unit, ScHARR, University of Sheffield; 2012. Available from: http://www. nicedsu.org.uk/PFSOS\%20Report.FINAL.06.08.12.pdf. Accessed March 30, 2012.

12. Saad ED, Katz A, Hoff PM, Buyse M. Progression-free survival as surrogate and as true end point: insights from the breast and colorectal cancer literature. Ann Oncol. 2010;21(1):7-12.

13. Olivo SA, Macedo LG, Gadotti IC, Fuentes J, Stanton T, Magee DJ. Scales to assess the quality of randomized controlled trials: a systematic review. Phys Ther. 2008;88(2):156-175.

14. Swinscow TDV, Campbell MJ. Statistics at Square One, ninth edition. Section 11. Correlations and regressions [webpage on the Internet]. London: BMJ Publishing Group Ltd; 1997. Available from: http://www. bmj.com/about-bmj/resources-readers/publications/statistics-squareone/11-correlation-and-regression. Accessed November 30, 2011.

15. Broglio KR, Berry DA. Detecting an overall survival benefit that is derived from progression-free survival. J Natl Cancer Inst. 2009;101(23):1642-1649. 
16. Cardoso F, Harbeck N, Fallowfield L, Kyriakides S, Senkus E; ESMO Guidelines Working Group. Locally recurrent or metastatic breast cancer: ESMO Clinical Practice Guidelines for diagnosis, treatment and follow-up. Ann Oncol. 2012;23 Suppl 7:vii11-vii19.

17. Hackshaw A, Knight A, Barrett-Lee P, Leonard R. Surrogate markers and survival in women receiving first-line combination anthracycline chemotherapy for advanced breast cancer. Br J Cancer. 2005;93(11): $1215-1221$.

18. Sherrill B, Amonkar M, Wu Y, et al. Relationship between effects on time-to-disease progression and overall survival in studies of metastatic breast cancer. Br J Cancer. 2008;99(10):1572-1578.

19. Miksad RA, Zietemann V, Gothe R, et al. Progression-free survival as a surrogate endpoint in advanced breast cancer. Int $J$ Technol Assess Health Care. 2008;24(4):371-383.

20. Burzykowski T, Buyse M, Piccart-Gebhart MJ, et al. Evaluation of tumor response, disease control, progression-free survival, and time to progression as potential surrogate end points in metastatic breast cancer. J Clin Oncol. 2008;26(12):1987-1992.
21. Matsubara Y, Sakabayashi S, Nishimura T, et al. Surrogacy of tumor response and progression-free survival for overall survival in metastatic breast cancer resistant to both anthracyclines and taxanes. Int J Clin Oncol. 2011;16(6):623-629.

22. Daniels MJ, Hughes MD. Meta-analysis for the evaluation of potential surrogate markers. Stat Med. 1997;16(17):1965-1982.

23. Gail MH, Pfeiffer R, Van Houwelingen HC, Carroll RJ. On metaanalytic assessment of surrogate outcomes. Biostatistics. 2000;1(3): 231-246.

24. Buyse M. Use of meta-analysis for the validation of surrogate endpoints and biomarkers in cancer trials. Cancer J. 2009;15(5): 421-425.

25. Buyse M, Molenberghs G, Burzykowski T, Renard D, Geys H. The validation of surrogate endpoints in meta-analyses of randomized experiments. Biostatistics. 2000;1(1):49-67.
OncoTargets and Therapy

\section{Publish your work in this journal}

OncoTargets and Therapy is an international, peer-reviewed, open access journal focusing on the pathological basis of all cancers, potential targets for therapy and treatment protocols employed to improve the management of cancer patients. The journal also focuses on the impact of management programs and new therapeutic agents and protocols on

\section{Dovepress}

patient perspectives such as quality of life, adherence and satisfaction The manuscript management system is completely online and includes a very quick and fair peer-review system, which is all easy to use. Visit http://www.dovepress.com/testimonials.php to read real quotes from published authors. 